\title{
Atenção em saúde mental para adolescentes femininas em Unidades Socioeducativas: dilemas de governança e medicalização
}

\author{
Mental health care for female adolescents in detention centres: \\ governance dilemmas and medicalization
}

Paulo Roberto Fagundes da Silva', Fabiana Lozano Gama², Nilson do Rosário Costa1

DOI: 10.1590/0103-11042019S705

\begin{abstract}
RESUMO O trabalho analisa a utilização de medicamentos psicoativos por adolescentes femininas internadas em Unidades Socioeducativas (USEs) no Brasil. Descreve as diretrizes da política pública nacional e a qualidade da coordenação da atenção em saúde mental pelos governos subnacionais. O artigo tem como hipótese que a governança verticalizada da USE está associada à utilização de medicação psicoativa. O modelo da pesquisa foi estudo comparado dos casos do Rio Grande do Sul, Minas Gerais e Rio de Janeiro. Os dados resultaram da coleta em prontuários e entrevistas com informantes-chave. $\mathrm{O}$ artigo demonstra que a governança vertical observada no Rio Grande do Sul está associada à alta prevalência de diagnósticos de transtorno mental e ao uso de medicamentos psicoativos pelas adolescentes privadas de liberdade. Na assistência verticalizada no Rio de janeiro, a adoção de um protocolo clínico em que o psiquiatra é o último profissional na linha de cuidado resultou no uso residual de psicofármacos. A despeito da articulação com atenção primária, a utilização de medicação psiquiátrica observada na USE feminina de Minas Gerais não era residual por alcançar $45 \%$ das adolescentes custodiadas. As evidências revelam que as sanções de privação de liberdade por atos infracionais podem produzir decisões de medicalização que ameaçam o direito dos adolescentes no campo da saúde. As diretrizes da política nacional incentivam escolhas mais cautelosas. As psicoterapias e as ações de reabilitação são as intervenções de primeira linha defendidas. A adoção da medicação deve incluir a avaliação dos riscos e benefícios. O manejo cuidadoso da intervenção psicofarmacológica pode evitar o excesso de prescrições e a subestimação dos efeitos adversos.
\end{abstract}

PALAVRAS-CHAVE Adolescente institucionalizado. Saúde mental. Psicotrópicos. Medicalização.

ABSTRACT The article analyzes the utilization of psychoactive drugs by young female offenders admitted to detention centres (USEs - Socio-Educational Unities) in Brazil. The article also describes how national public agencies' guidelines and the quality of coordinated action is used by subnational instances regarding mental health care. This article results from three case studies in Rio Grande do Sul, Minas Gerais and Rio de Janeiro. The central hypothesis of the research is that Youth Detention Center's governance regime could explain psychiatric medicalization preferences. The cross-sectional data resulted from medical records and interviews with key informants. It shows that vertical governance observed in Rio Grande do Sul is directly associated with high prevalence of diagnosis of mental health disorder and psychoactive drugs utilization by young offenders who are deprived of freedom. In the model of vertical governance promoted in Rio de Janeiro, the adoption of a clinical protocol in which the psychiatrist is the last professional in the line of care resulted in a residual use of psychiatric medication. Despite the articulation with primary care, the use of psychiatric medication observed in Minas Gerais was not residual because it reached $45 \%$ of the adolescents in custody. These findings indicate that sanctions of imprisonment for illegal acts may produce a set of medicalization decisions that threaten the rights of adolescents in detention centres in the field of health. Paradoxically, national mental health policies for young offenders proposes a more cautious approach in relation to psychiatric medication. National policies also sustains that medication should include an ongoing assessment of risks and benefits. The careful administration of psychopharmacological intervention can avoid excessive prescription and underestimation of adverse effects.

2 Secretaria Municipal de Saúde (SMS) - Rio de Janeiro (RJ), Brasil.

KEYWORDS Institutionalized adolescent. Mental health. Psychotropic drugs. Medicalization. 


\section{Introdução}

Este artigo descreve os arranjos de cooperação federativa e os modelos de atenção em saúde mental destinados às adolescentes internadas em Unidades Socioeducativas (USEs) nos estados do Rio Grande do Sul, Minas Gerais e Rio de Janeiro. O trabalho analisa também a prescrição e uso de psicofármacos, a prevalência dos diagnósticos psiquiátricos e a articulação das USEs com a Rede de Atenção Psicossocial (Raps) do Sistema Único de Saúde (SUS). As USEs são administradas por governos regionais em um contexto de descentralização federativa da assistência à saúde mental no âmbito do SUS'.

Com o processo de redemocratização da década de 1980, os indivíduos com transtorno mental e a população infantojuvenil encarcerada foram favorecidos pela legislação orientada aos direitos civis e sociais: o Estatuto da Criança e do Adolescente (ECA, Lei no $\left.{ }^{\circ} .069 / 90\right)$ e a Lei da Reforma Psiquiátrica (LRP, Lei $\mathrm{n}^{\circ}$ 10.216/2001). Desde então, ao adolescente autor de atos infracionais, são aplicadas as Medidas Socioeducativas (MSE), sendo a internação em estabelecimento educacional a decisão em última instância e a mais severa ${ }^{2}$.

Para a internação de adolescente em estabelecimento socioeducativo, o ECA estabelece a brevidade, a excepcionalidade e o respeito à condição peculiar de pessoa em desenvolvimento, considerando a gravidade do ato infracional, a reincidência de infrações graves ou o descumprimento reiterado da MSE anteriormente imposta. Em relação à saúde mental, o ECA indica, genericamente, que o tratamento deve ser individual e realizado em local adequado às condições do adolescente custodiado.

Ao portador de transtorno mental, a internação de longa permanência em hospital psiquiátrico deixou de ser o padrão de referência na esfera pública. A LRP determina que a internação seja indicada apenas quando esgotados os recursos extra-hospitalares, em especial o Centro de Atenção Psicossocial (Caps), referência comunitária e territorial para a assistência em saúde mental em substituição ao modelo asilar de tratamento ${ }^{3}$.

A proposta para Sistema Nacional de Atendimento Socioeducativo (Sinase), contida na Lei $\mathrm{n}^{0} 12.594$, de 2012, foi mais longe na articulação entre o ECA e a LRP ao definir que a assistência ao adolescente com transtorno mental deve acompanhar às orientações da LRP, privilegiando os dispositivos extra-hospitalares do SUS4 ${ }^{4}$ Ainda que tardiamente, o Sinase promove o completo alinhamento da assistência em saúde do adolescente privado de liberdade com as novas diretrizes da saúde mental, propondo o direito de tratamento em serviços comunitários de saúde mental2 ${ }^{2}$.

A Política Nacional de Atenção Integral à Saúde de Adolescentes em Conflito com a Lei, inaugurada em 2004 e atualizada em 2010 e 2014 (PNAISARI - Portaria $n^{0} 1.082 / 2014$ ), ampliou o alinhamento da ECA com o SUS ao incentivar a atenção em saúde mental do adolescente submetido à medida socioeducativa nas três esferas de governo. O plano operativo proposto pela PNAISARI contempla diretrizes e ações na atenção básica, média e alta complexidade e define as responsabilidades de cada esfera de gestão no financiamento e provisão ${ }^{2}$.

Apesar desses avanços normativos, o risco da medicalização nas organizações socioeducativas não esteve no centro das preocupações, e ainda há uma grande lacuna sobre a compreensão do componente farmacológico, incluindo o uso e o manejo de psicofármacos. A medicalização foi incluída na agenda pública brasileira muito recentemente por meio da Resolução ${ }^{\circ}$ 177, de 2015, do Conselho Nacional dos Direitos da Criança e do Adolescente (Conanda), que dispõe sobre o direito da criança e do adolescente de não serem submetidos à excessiva medicalização e recomenda protocolos clínicos que privilegiem alternativas não medicalizantes, considerando aspectos pedagógicos, sociais, culturais, emocionais e étnicos que envolvam trabalhos com a família, profissionais e comunidade, por meio de abordagem multiprofissional e intersetorial, promovendo práticas de educação e promoção da saúde 5 . 
Conrad descreve a medicalização como um processo no qual problemas não médicos são traduzidos e apropriados como problemas médicos por meio de diagnósticos e imposição de tratamento ${ }^{6}$. A literatura identifica uma vasta gama de problemas humanos que ingressaram na jurisdição médica - desde os desvios comportamentais até os eventos triviais, como o nascimento, a menopausa e o envelhecimento7.

Estudos recentes têm identificado o componente farmacêutico da medicalização, denominando-o de farmaceuticalização. O termo designa o processo pelo qual condições sociais, comporta-mentais e corporais são tratadas ou consideradas com necessidade de tratamento medicamentoso por médicos, pacientes ou ambos ${ }^{\mathbf{8}, \mathbf{9}}$. Cabe aqui chamar atenção que a produção intelectual sobre a medicalização e a farmaceuticalização raramente descreve os efeitos sobre organizações e profissões desses processos. Na profissão médica, por exemplo, a ampliação do escopo e da efetividade dos novos medicamentos restaurou a posição normativa e o valor das especialidades, sendo a especialidade psiquiátrica uma das mais beneficiadas. A prescrição dos psicofármacos constitui uma das ferramentas cruciais da atuação clínica dos psiquiatras contemporâneos no ambiente organizacional e no mercado de prestação de serviços ${ }^{\mathbf{1 0}}$.

A experiência brasileira da medicalização nas USEs é relevante por permitir a compreensão dos afeitos de diretrizes normativas sobre o padrão da assistência à saúde mental de viés medicalizante e farmacológico em prisões e organizações de custódia. Em razão do contexto organizacional em que é operada, a assistência à saúde mental das USEs é também subordinada às preferências profissionais, especialmente de psiquiatras e psicólogos. Como indica a literatura do campo, as organizações orientadas por mecanismos profissionais permitem elevada autonomia decisória às profissões que detêm monopólio de competências reconhecido por lei1․

Diante disso, este trabalho adota o pressuposto que as pressões administrativas e legais podem afetar a autonomia decisória das profissões de saúde nas USEs, especialmente daquelas que operam por meio da provisão de serviços de saúde mental de modo verticalizado. Em desafio ao modelo verticalizado, pressões administrativas e legais desencadeadas pelo Sinase incentivam a cooperação com os municípios na assistência à saúde da população adolescente sob custódia ${ }^{1}$. A orientação para a cooperação contemplaria o privilegiamento dos dispositivos comunitários de assistência à saúde mental e a inibição de decisões profissionais voltadas para opções de tratamento medicalizantes ${ }^{2}$.

Este artigo traz à luz a presença da população adolescente feminina em regime de privação de liberdade no ambiente organizacional socioeducativo. Cabe assinalar que a condição das adolescentes em conflito com a lei é registrada modo superficial nos documentos oficiais brasileiros ${ }^{\mathbf{1 2}}$. Especialmente em relação à assistência à saúde mental, a ausência da questão de gênero no ambiente socioeducativo é particularmente dramática apesar do mal-estar que a situação de encarceramento pode produzir nas adolescentes.

Os estudos nacionais e internacionais sobre a prevalência de transtornos mentais vis- $\dot{a}$-vis privação de liberdade verificaram que $70 \%$ dos adolescentes masculinos podem ser diagnosticados com transtornos mentais ${ }^{\mathbf{1 3}}$. Porém, para a população adolescente feminina, a prevalência de transtornos mentais na mesma situação pode ser significativamente mais alta, como sustentam os trabalhos de Karnik et al. (2009) ${ }^{14}$ : 98\%, Pinho et al. (2006) ${ }^{\mathbf{1 5}}$ : 75\%; e Verugdenhil et al. (2004) ${ }^{\mathbf{1 6}}$ : 90\%. Mesmo com a cautela necessária em relação à tendência à inflação diagnóstica desses estudos, a diferença da prevalência de transtornos mentais na situação de internação de adolescentes femininas justifica que a compreensão da atenção em saúde mental no ambiente socioeducativo pelo viés da condição de gênero seja incluída na agenda de pesquisa.

Este artigo reconhece que a relação entre gênero e violência permanece com muitas 
questões em aberto. Esbensen e colaboradoras apontam, nesse sentido, que o crescimento nas últimas três décadas da preocupação com o envolvimento feminino na violência exige a revisão da perspectiva teórica tradicional de viés masculino sobre o tema. Segundo os autores, é necessário ampliar as respostas sobre os problemas e eventos associados ao conflito com a lei na adolescência. Esses eventos são neutros às questões de gênero? Meninos e meninas conflitam com a lei por motivações ou causas diferentes? Caso existam discrepâncias significativas de gênero nas motivações de violência e nos efeitos sobre as condições de saúde mental, os autores sugerem que diferentes abordagens assistenciais sejam desenvolvidas ${ }^{17}$.

\section{Desenho do estudo}

Considerando essas preocupações teóricas, este artigo apresenta o resultado da pesquisa exploratória e transversal realizada nas cidades de Porto Alegre (2014), Belo Horizonte (2015) e Rio de Janeiro (2016-2017) nas USEs estaduais de internação. Adotou-se a estratégia do estudo de casos para descrever e analisar a atenção à saúde mental nessas organizações de custódia. O desenho de estudo de casos múltiplos facultou a formulação de questões gerais para orientar o exame de cada caso ${ }^{\mathbf{1 8}}$. A comparação controlada de um pequeno número pôde também orientar a coleta sistemática de dados e a coleta dos componentes da mesma variável em cada unidade (caso).

Foram consultados 20 prontuários na unidade de Porto Alegre, 42 na unidade de Belo Horizonte e 35 na unidade do Rio de Janeiro. Nesses prontuários, estavam disponíveis as informações da totalidade das adolescentes internadas nas respectivas USEs nos dias das visitas das equipes de pesquisa (excluídas as adolescentes em situação de internação provisória). As informações foram coletadas dos prontuários por meio de instrumento estruturado.
A coleta de informações primárias sobre o uso de medicação foi realizada pela consulta nas fichas de controle de prescrição médica no setor de enfermagem, que informava o tipo de medicação que a adolescente utilizava no dia da visita da equipe de pesquisa, e pela análise das informações dos prontuários sobre a medicação. A pesquisa coletou, portanto, a informação primária sobre a atenção em saúde mental diretamente nas USEs.

Foram também utilizadas entrevistas abertas com informantes-chave selecionados de forma intencional a partir de avaliações ocorridas em cada município do estudo: três gestores do sistema socioeducativo e três médicos psiquiatras que prestam atendimento aos adolescentes internados nas USEs (um de cada caso estudado nas duas categorias de entrevistados).

Em Porto Alegre, foi pesquisado o Centro de Atendimento Socioeducativo Feminino (Case), que é responsável pelo atendimento de adolescentes do sexo feminino que cumprem medida de semiliberdade, Internação Com Possibilidade de Atividade Externa (ICPAE), Internação Sem Possibilidade de Atividade Externa (Ispae) e internação provisória, oriundas dos dez Juizados da Infância e da Juventude do Rio Grande do Sul. Em Belo Horizonte, o Centro de Reeducação Social São Jerônimo (CRSSJ), vinculado à Subsecretaria de Atendimento às Medidas Socioeducativas, vinculada à Secretaria de Estado de Defesa Social. Na cidade do Rio de Janeiro, o Centro Socioeducativo Professor Antônio Carlos Gomes da Costa (PACGC) que é a única USE destinada à execução da MSE de internação e internação provisória para adolescentes do sexo feminino no estado. O PACGC é vinculado ao Departamento Geral de Ações Socioeducativas (Degase) da Secretaria de Estado de Educação.

Como já assinalado, o artigo tem como pressuposto que a adoção de condutas medicalizantes pode estar associada à provisão de serviços em saúde mental exclusivamente no interior da USE a despeito das orientações do 
Sinase. Nesse sentido, o padrão da organização da assistência em saúde mental e a alta prevalência do uso de psicofármacos justificam a análise dos dispositivos socioeducativos. A baixa frequência de utilização da medicação psiquiátrica é considerada como um bom indicativo da adequação da assistência em saúde mental nas USEs.

Os dados foram analisados por meio de estatística descritiva em ambiente SPPS 24.0. A pesquisa foi aprovada pelo Comitê de Ética em Pesquisa da Escola Nacional de Saúde Pública Sergio Arouca da Fundação Oswaldo Cruz (CAAE: 30474514.1.0000.5240).

\section{Resultados}

O perfil social das adolescentes em conflito com a lei nos estados pouco difere do padrão geral dos adolescentes privados de liberdade do País: baixíssima escolaridade - 87\% com fundamental incompleto. A alta proporção das adolescentes com fundamental incompleto revela que a experiência da evasão escolar é muito expressiva no grupo social.
As adolescentes também eram predominantemente categorizadas institucionalmente como de 'raça' negra ou parda (58\%) e com idade mediana bastante próxima à maioridade $(17,5)$.

Em relação ao tipo de ato infracional, prevalecem os graves (40\%), seguidos pelo roubo (32\%) e tráfico de drogas (18\%). Segundo a Súmula 492 do Supremo Tribunal de Justiça ${ }^{19}$, os atos considerados mais graves são aqueles contra a vida: homicídio, latrocínio e roubos com violência (qualificado). O tráfico de drogas e a receptação são considerados de menor gravidade.

A tabela 1 demonstra que $20 \%$ das adolescentes (19 do total de 99) eram reincidentes. Entre as adolescentes na condição de reincidente, $68 \%$ foram privadas de liberdade pelo mesmo ato infracional leve (tráfico de drogas e roubo). A penalização da reincidência revela a preferência do Judiciário dos estados estudados pela decisão de privação de liberdade mesmo quando o ato infracional leve é reiterado pelo adolescente. De qualquer modo, é importante sublinhar, ainda assim, a proporção de adolescentes mulheres privadas de liberdade em razão de delitos graves (40\%) na população estudada neste artigo.

Tabela 1. Condição social, delitos e assistência em saúde mental das adolescentes em privação de liberdade em Porto Alegre, Belo Horizonte e Rio de Janeiro. 2014, 2015, 2016 (N=99)

\begin{tabular}{lcr}
\hline Descritor & Frequência & $\%$ \\
\hline Idade acima de 17,5 anos & 50 & $50 \%$ \\
Ensino Fundamental incompleto & 86 & $87 \%$ \\
Categorizadas como 'raça' negra ou parda & 57 & $58 \%$ \\
Reincidentes & 19 & $20 \%$ \\
Delitos graves (homicídio, latrocínio e roubo qualificado) & 38 & $40 \%$ \\
Delito leve (tráfico) & 32 & $32 \%$ \\
Delito leve (roubo) & 18 & $18 \%$ \\
Adolescentes em tratamento em saúde mental & 60 & $61 \%$ \\
Adolescentes que usaram medicação durante a internação & 44 & $44 \%$ \\
Adolescentes com um ou mais diagnóstico & 29 & $29 \%$ \\
Adolescentes sem diagnóstico & 70 & $71 \%$ \\
Em tratamento apenas nos serviços de saúde mental da USE & 41 & $41 \%$ \\
Em tratamento em serviços de saúde mental do SUS & 19 & $20 \%$ \\
\hline Fonte: Pesquisa 'Diagnóstico situacional da atenção em saúde mental nas unidades socioeducativas brasileiras'. DCS/Ensp/Fiocruz.2016/2017.
\end{tabular}


Com base nos dados do Rio de Janeiro ( $\mathrm{n}=35)$, é possível observar que os atos infracionais considerados mais graves, como homicídio e violência armada, estavam associados a maior tempo de internação (gráfico 1). No caso do homicídio, $50 \%$ dos casos estavam em privação de liberdade entre 50 dias e 250 dias, com mediana em aproximadamente 180 dias. As adolescentes situadas no quartil superior permaneceram internadas entre 250 dias e 370 dias aproximadamente. Os atos infracionais relacionados com sequestro e violência armada, como o latrocínio, por exemplo, ocuparam também tempos maiores de encarceramento. O tempo mínimo foi de aproximadamente 100 dias; e o máximo, de 381 dias. Nesse sentido, pode-se verificar que o tempo de internação possui relação com a gravidade do ato infracional praticado. Os pontos mais afastados do gráfico representam as adolescentes julgadas por atos infracionais leves e reincidentes, corroborando também a associação entre reincidência, internação e penas longas no estado do Rio de Janeiro.

Gráfico 1. Tempo de internação e categoria do ato infracional das adolescentes privadas de liberdade no estado do Rio de Janeiro

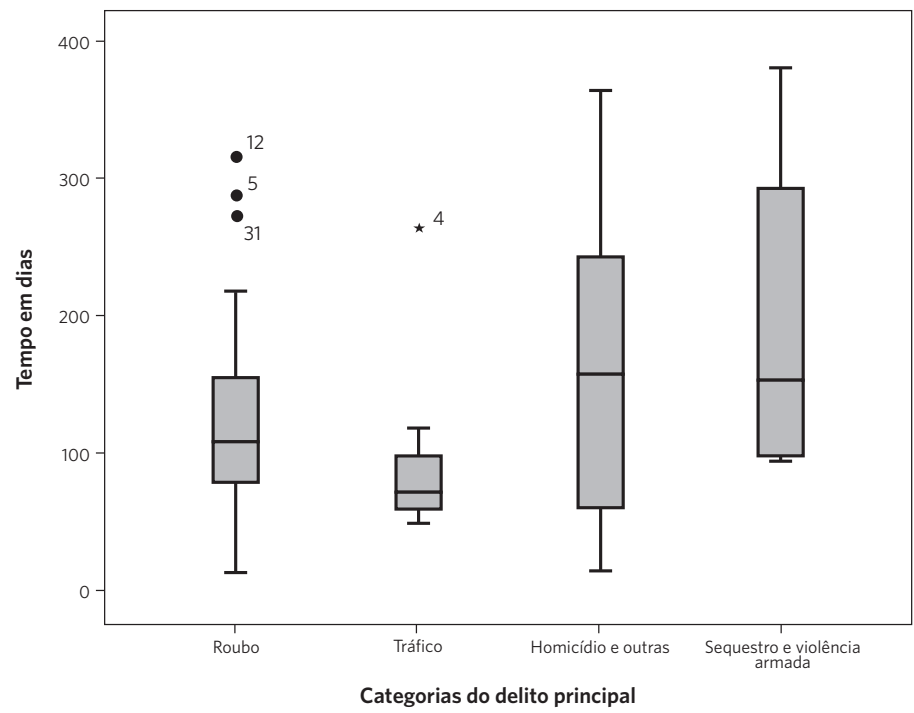

Fonte: Pesquisa 'Diagnóstico situacional da atenção em saúde mental nas unidades socioeducativas brasileiras'. DCS/Ensp/Fiocruz. 2016/2017.

A tabela 1 mostra a proporção das adolescentes em privação de liberdade em tratamento de saúde mental nas USEs dos três estados (61\%). A pesquisa considerou como 'tratamento em saúde mental' tanto o uso de medicação psiquiátrica quanto outras modalidades de atenção, como, por exemplo, os atendimentos psicológicos ou a participação em oficinas terapêuticas, consideradas intervenções psicossociais. $\mathrm{O}$ uso de psicofármacos era elevado ( $44 \%$ das adolescentes utilizaram medicação durante a internação). A frequência de adolescentes em tratamento e usando medicação psiquiátrica contrasta com a também expressiva proporção de adolescente nas três cidades sem diagnóstico (70\%).

A análise do padrão de integração com a rede de saúde do SUS e, portanto, do nível de 
aderência às diretrizes do Sinase revela que os estados estudados optavam por diferentes padrões de verticalização da gestão dos serviços de saúde com importantes repercussões sobre o modelo assistencial seguido.

Como mostra a tabela 2, na USE estadual no município de Porto Alegre, a opção predominante de gestão foi pela verticalização da assistência à saúde mental. De um total de 20 adolescentes, 18 (90\%) estavam em tratamento em saúde mental nos serviços próprios da USE durante a internação, sendo que 13 (65\%) fizeram uso de psicofármacos, e 5 realizaram intervenções psicossociais. A característica importante do modelo assistencial do Rio Grande do Sul era a presença de psiquiatras contratados por cooperativas médicas nas USEs, sendo que a avaliação psiquiátrica era uma das primeiras intervenções realizadas. Os psicólogos internos se dedicavam a gestão dos delitos no âmbito da ressocialização, oferendo intervenções breves em relação ao projeto de vida da adolescente. Não ofereciam terapias ou grupos, carecendo de alternativas de atenção psicossocial2 ${ }^{2}$. A baixa adoção de intervenções psicossociais na unidade socioeducativa de Porto Alegre, além da escassez da oferta em serviços comunitários em saúde mental (utilizados por duas adolescentes) e de atenção básica, poderia explicar a alta frequência de adolescentes em uso de psicofármacos.

Na USE estadual situada em Belo Horizonte, 27 (64\%) das adolescentes internadas estavam em tratamento em saúde mental, e 19 (45\%) estavam em uso de psicofármacos. O governo estadual optou por uma gestão compartilhada nas intervenções em saúde mental: a maioria das adolescentes em tratamento frequentavam dispositivos externos municipais ou privados (67\%). Entre os dispositivos externos, a Atenção Básica em Saúde teve participação importante no atendimento de $33 \%$ dos adolescentes. Nesse contexto, foi observado que a articulação do sistema socioeducativo ultrapassou os limites da interação com a rede de saúde mental municipal, incluindo a Estratégia Saúde da Família (ESF). A despeito dessa articulação com atenção primária, a utilização de medicação psiquiátrica observada na USE feminina de Minas Gerais não era residual por alcançar $45 \%$ das adolescentes custodiadas. Resultados de pesquisas não autorizam a tese de uma orientação menos medicamentosa na atenção primária. Frances ${ }^{20}$ considera que a expansão do conceito de transtorno mental (o que ele denomina inflação diagnóstica) vem acarretando um indesejável aumento no consumo de psicotrópicos nos Estados Unidos, sendo que $80 \%$ das prescrições são feitas por médicos da atenção primária com pouco treinamento e tempo insuficiente para fazer um diagnóstico acurado.

No caso do Rio de Janeiro, observa-se, pela tabela 2, que nenhuma opção de assistência em saúde mental em dispositivos externos do SUS estava disponível para as adolescentes internadas no PACGC ao contrário dos demais estados. O levantamento nos prontuários revelou que a assistência em saúde mental era concentrada exclusivamente nos serviços próprios da USE. O Degase preservava integralmente a governança verticalizada da atenção em saúde mental a despeito das normativas legais. Diante dessa evidência, poder-se-ia esperar que a medicalização fosse predominante nas decisões de tratamento do PACGC. Entretanto, apesar do modelo verticalizado, apenas sete das adolescentes (19\%) tinham registro de uso de medicamento psiquiátrico durante a internação. Na unidade, 15 adolescentes (40\%) estavam também em tratamento em saúde mental. 
Tabela 2. Modelo de governança e a articulação do tratamento em saúde mental com o Sistema Único de Saúde em Unidades Socioeducativas $(\mathrm{N}=99)$

\begin{tabular}{lrrrr}
\hline Modelo de Governança / Adolescentes em & Porto Alegre & Belo Horizonte & Rio de Janeiro & Total \\
Tratamento nos Estados & 16 & 9 & 15 & 40 \\
\hline Tratamento Exclusivamente em Serviços Próprios & 2 & 18 & 0 & 20 \\
Tratamento com o SUS & 2 & 15 & 22 & 39 \\
Sem Tratamento & 20 & 42 & 37 & 99 \\
Total & & & 39 \\
\hline
\end{tabular}

Fonte: Pesquisa 'Diagnóstico situacional da atenção em saúde mental nas unidades socioeducativas brasileiras'. DCS/Ensp/Fiocruz. 2016/2017.

Ao contrário do modelo adotado em Belo Horizonte, as entrevistas evidenciaram que o Rio de Janeiro privilegiou a implantação de protocolos clínicos para responder indiretamente às exigências normativas do Sinase e às recomendações do Conanda sem promover a integração com os dispositivos do SUS da cidade do Rio de Janeiro. Foi estruturada uma equipe multiprofissional especializada em saúde mental no âmbito das USEs denominado 'Núcleo de Saúde Mental' que promove a prevenção de agravos em saúde mental durante a internação. A linha de cuidado contempla atendimentos individuais e em grupo, realização e organização de grupos temáticos, oficinas terapêuticas, grupos de prevenção, grupos de familiares ou responsáveis e atividades externas com os adolescentes. Segundo esse protocolo, o psiquiatra é o último profissional a avaliar o adolescente e atua nos casos em que todas as demais intervenções psicossociais foram esgotadas ${ }^{21}$.

A comparação entre as USEs estaduais sugere que a presença do psiquiatra no ambiente institucional sem a implantação concomitante de protocolos clínicos e a devida integração com equipes multiprofissionais podem ser associadas à alta prevalência dos diagnósticos psiquiátricos da população feminina custodiada. Como mostra a tabela 3 , na unidade do Rio Grande do Sul, $75 \%$ das adolescentes tinham registro de diagnóstico psiquiátrico na USE Case. Essa proporção contrasta especialmente com a prevalência residual de $11 \%$ observada na unidade do estado do Rio de Janeiro. Na unidade São Jerônimo de Minas Gerais, a proporção constada de $26 \%$ era também inferior ao observado no Rio Grande do Sul, embora não tão residual como constatada no Rio de Janeiro.

Tabela 3. Diagnósticos psiquiátricos em prontuários - Unidades Socioeducativas de Belo Horizonte, Porto Alegre e Rio de Janeiro

\begin{tabular}{lrrr}
\hline Unidades & $\begin{array}{r}\text { Total de Prontuários } \\
\text { (A) }\end{array}$ & $\begin{array}{r}\text { Prontuários com 1 ou mais } \\
\text { Diagnósticos (B) }\end{array}$ & B/A*100 \\
\hline São Jerônimo (Belo Horizonte) & 42 & 11 & $26 \%$ \\
Case (Porto Alegre) & 20 & 15 & $75 \%$ \\
PACGC (Rio De Janeiro) & 37 & 4 & $11 \%$ \\
Total & 99 & 30 & $30 \%$ \\
\hline
\end{tabular}

Fonte: Pesquisa 'Diagnóstico situacional da atenção em saúde mental nas unidades socioeducativas brasileiras'. DCS/Ensp/Fiocruz. 2016. 
A tabela 4 mostra a associação positiva entre o diagnóstico formal do transtorno mental no prontuário das adolescentes nos estados e o registro de utilização medicação psiquiátrica. Essa associação era fortemente influenciada pelas informações dos prontuários na unidade
Case do Rio Grande do Sul, como já comentado. A proporção das adolescentes que utilizaram medicamentos psicoativos durante a internação variou expressivamente de 19\% no Rio de Janeiro, a 45\% em Minas Gerais, e a $90 \%$ no Rio Grande do Sul.

Tabela 4. Condição diagnóstica e prescrição medicamentosa em prontuários - Unidades Socioeducativas de Porto Alegre, Belo Horizonte e Rio de Janeiro

\begin{tabular}{lrrr}
\hline Diagnóstico/Medicação & Sem medicação & Com medicação & Total \\
\hline Sem diagnóstico no prontuário & 52 & 18 & 70 \\
Com diagnóstico no prontuário & 3 & 26 & 29 \\
Total & 55 & 44 & 99 \\
\hline
\end{tabular}

Fonte: Pesquisa 'Diagnóstico situacional da atenção em saúde mental nas unidades socioeducativas brasileiras'. DCS/Ensp/Fiocruz. 2016.

A terceira coluna da tabela 4 mostra a surpreendente frequência de adolescentes que utilizavam medicamento psiquiátrico sem informação do diagnóstico no prontuário (quatro em cada dez adolescentes em uso de medicação). Essa frequência estava longe de ser residual, especialmente na USE feminina de Minas Gerais. A mesma situação foi observada na unidade do Rio de Janeiro. A explicação para inexistência de diagnóstico no prontuário pode ser pensada de modo positivo pela tentativa de redução do estigma associado aos diagnósticos de transtornos mentais, evitando a rotulação. A influência da psicanálise nas condutas de tratamento, especialmente em Minas Gerais, também pode explicar a inconsistência informacional dos prontuários. A psicanálise tem uma visão sobre o diagnóstico mais estrutural do que fenomenológica, logo, as categorias diagnósticas clássicas da psiquiatria perdem a sua significação ${ }^{22}$. É necessário, ainda assim, considerar como problema não trivial na orientação de tratamento das adolescentes custodiadas nos dois Estados que a prescrição medicamentosa pressupõe a existência de uma hipótese diagnóstica que indique clinicamente o uso do psicofármaco.

Em relação à medicação utilizada no momento da visita da equipe de pesquisa, foram analisadas 36 prescrições, sendo 17 do Case, 13 do São Jerônimo e 6 do PACGC. Utilizavam ansiolíticos 10 adolescentes (10,1\%); antidepressivos, 8 (8,1\%); metilfenidato, 9 (9,1\%); antipsicóticos, 11 (11,1\%); estabilizadores do humor, 3 (3\%) e anticolinérgicos, 2 (2\%).

Em relação aos antidepressivos, seu uso nas chamadas depressões menores pode ser uma escolha inadequada. Revisão publicada em 2011 não evidenciou vantagens clínicas na comparação entre os antidepressivos e placebos no manejo das depressões menores. Existe clara indicação dos benefícios dos tratamentos psicológicos para esses quadros, o que sugere que os antidepressivos não devem ser considerados para o tratamento inicial dos indivíduos. Em relação ao uso de ansiolíticos, é necessário ponderar os potenciais benefícios de sua rápida eficácia com os riscos de uso indevido, dependência e sintomas de abstinência ${ }^{23}$.

O metilfenidato tem sido a primeira opção terapêutica para o Transtorno de Deficit de Atenção, com ou sem Hiperatividade (TDA/H) em crianças e adultos. Seu uso é objeto de controvérsias principalmente porque é também utilizado para melhoramento do desempenho cognitivo de indivíduos saudáveis ${ }^{24}$. 
As entrevistas qualitativas com psiquiatras indicam que o alto uso de antipsicóticos nas unidades pode estar relacionado com o manejo clínico da impulsividade. Porém, alguns autores consideram que inexiste evidência clínica (por ensaios clínicos) para o uso de antipsicóticos e outras medicações como inibidores seletivos da serotonina no tratamento de impulsividade. Os protocolos clínicos indicam a intervenção psicossocial como primeira opção, e, no caso de uso de medicação, dirigi-la para o manejo do transtorno mental primário mais do que ao enfrentamento específico da agressividade ${ }^{25}$.

\section{Considerações finais}

O modelo de governança da assistência em saúde mental observado nas USEs femininas do Rio Grande do Sul e do Rio de Janeiro era verticalizado, indicando que, no momento da pesquisa, persistia nos estados o conflito normativo com as diretrizes da política pública nacional. Nas USEs femininas, as relações de cooperação dessas unidades com a rede municipal de serviços comunitários e com a atenção primária eram residuais ou nulas.

Nesses dois estados, a governança verticalizada desenvolveu modalidades de organização da assistência em saúde mental ancoradas nas preferências das profissões de saúde, ainda que com resultados substancialmente divergentes nas decisões de diagnóstico e tratamento com base na medicação.

No Rio Grande do Sul, o modelo de governança verticalizado privilegiava às condutas clínicas farmacológicas da psiquiatria. Observa-se na USE feminina do estado à inflação diagnóstica e a prescrição em alta escala de psicofármacos. No estado, nove em cada dez adolescentes femininas utilizavam medicação psiquiátrica no momento da pesquisa. Estudo anterior já demonstrara que as unidades masculinas do estado apresentavam também o mesmo perfil de verticalização, inflação diagnóstica e prescrição de psicofármacos ${ }^{26}$. Comparando os dois estudos, pode-se afirmar que as decisões de medicalização afetaram do mesmo modo tanto a população masculina quanto a feminina, não sendo observada qualquer diferença significativa da condição de gênero.

Esse padrão assistencial observado no Rio Grande do Sul conflita com as orientações da literatura. A inflação diagnóstica e o uso intensivo de psicofármacos na população privada de liberdade retiraram de cena as múltiplas causalidades que estão em jogo na situação infracional com privação de liberdade, impedindo a leitura ampliada da vulnerabilidade social dos adolescentes. Ademais, a rotulação causada pelo diagnóstico de transtorno mental favorece a desresponsabilização do sujeito ante $\mathrm{o}$ ato infracional independentemente da condição de gênero ${ }^{27}$.

$\mathrm{Na}$ assistência verticalizada do estado do Rio de Janeiro, a adoção de um protocolo clínico - em que o psiquiatra é o último profissional na linha de cuidado - resultou no uso residual de psicofármacos (apenas duas em dez adolescentes usavam medicação no momento da pesquisa). Ainda assim, o Degase mantinha as intervenções em saúde mental intramuros para contornar as dificuldades organizacionais e operacionais de cooperação com os dispositivos comunitários municipais.

Para o gestor entrevistado, as condições dos serviços municipais na cidade do Rio de Janeiro não garantiam o acompanhamento sistemático e permanente das adolescentes custodiadas. Diante dessa percepção, o Degase definiu a organização verticalizada da assistência em saúde mental por equipe interna e propunha oferecer várias atividades psicossociais com o objetivo de reduzir o uso de psicofármacos para o manejo do sofrimento psíquico.

Os recursos externos e compartilhados eram utilizados apenas para os casos de emergência ou casos graves de transtornos primários. Cabe ressaltar que, nas entrevistas realizadas, os informantes-chave no Rio de Janeiro destacaram que a crise fiscal do estado no biênio 
2016-2017 já afetava severamente a governança da assistência à saúde mental verticalizada do Degase, repercutindo diretamente na disponibilidade de insumos para os adolescentes em uso de medicação psiquiátrica.

O caso da unidade pesquisada no estado de Minas Gerais destaca o ativismo da rede municipal de saúde na interação com o sistema socioeducativo. Costa e Silva apontam que a cooperação horizontal observada no estado evidencia algumas opções na direção de uma reforma da atenção em saúde mental que considere o direito da criança e do adolescente de não serem submetidos à excessiva medicalização e que privilegie a promoção da saúde ${ }^{2}$. No caso do estado do Rio de Janeiro, a existência de um protocolo clínico no ambiente organizacional do Degase expressa a decisão de ajustar as condutas organizacionais na assistência em saúde mental às diretrizes da política pública nacional. Contudo, a experiência da cooperação horizontal entre estado e municípios em Minas Gerais introduz instrumentos de articulação entre governos e organizações que podem tornar as escolhas assistenciais dos gestores mais abrangentes e sustentáveis.

Ainda que as diretrizes do PNAISARI para a articulação entre instâncias federativas sejam relativamente recentes, as ações de matriciamento entre a saúde municipal e o sistema socioeducativa devem ser encorajadas. Não resta dúvida que a disponibilidade do governo municipal à cooperação é um elemento crucial do processo de reforma da assistência à saúde mental no sistema socioeducativo brasileiro.

\section{Colaboradores}

Silva PRF (0000-0003-0811-4080)*, Gama FL (0000-0002-6916-0323)*, Costa NR (00000002-8360-4832)* contribuíram igualmente na elaboração do manuscrito. 


\section{Referências}

1. Costa NR, Siqueira SV, Uhr D, et al. A Reforma Psiquiátrica, Federalismo e Descentralização da saúde pública no Brasil. Ciênc. Saúde Colet, 2011; 16(12):46034614 .

2. Costa NR, Silva PRF. A atenção em saúde mental aos adolescentes em conflito com a lei no Brasil. Ciênc. Saúde Colet. 2017; 22(5):1467-1478.

3. Alves DSN, Silva PRF, Costa NR. Êxitos e Desafios da Reforma Psiquiátrica no Brasil. 22 Anos Após a Declaração de Caracas. Medwave. 2012; 12(10):45-55.

4. Brasil. Lei $\mathrm{n}^{\circ} 12.594$, de 18 de janeiro de 2012. Instituiu o Sistema Nacional de Atendimento Socioeducativo (SINASE), regulamenta a execução das medidas socioeducativas destinadas a adolescente que pratique ato infracional. Diário Oficial da União 2012; 5 Jan 2012. Brasil. Conselho Nacional de Justiça (CNJ). Panorama Nacional. A Execução de Medida Socioeducativa de Internação. Brasília, DF: CNJ; 2012.

5. Brasil. Presidência da República. Secretaria Especial dos Direitos Humanos. Sistema Nacional de Atendimento Socioeducativo (SINASE). Brasília, DF: CONANDA; 2015.

6. Conrad P. The medicalization of society: on the transformation of human conditions into treatable disorders. Baltimore: The John Hopkins University Press; 2007.

7. Conrad P, Schneider J. Deviance and medicalization: from badness to sickness. Philadelphia: Temple University Press; 1992.

8. Bell SE, Figert AE. Medicalization and Pharmaceuticalization at the intersections: a commentary on Bell and Figert. Soc Sci Med 2012; 75(5):775-783.

9. Abraham J. Pharmaceuticalization of Society in Context: Theoretical, Empirical and Health Dimensions. Sociology 2010; 44(4):603-622.

10. Rose N. Historical Change in Mental Health Practice.
In: Thornicroft G, Szmukler G, Mueser KT, et al., editores. Oxford Textbook of Mental Health. Oxford: Oxford University Press, 2011. p. 9-17.

11. Di Maggio PJ, Powell WW. The iron cage revisited: Institutional isomorphism and collective rationality in organization fields. In: Di Maggio PJ, Powell WW, editores. The New Institutionalism in Organizational Analysis. Chicago: The University of Chicago Press; 1991. p. 63-82.

12. Brasil. Secretaria Geral da Presidência da República. Mapa do Encarceramento. Brasília, DF: Secretaria Geral da Presidência da República; 2014.

13. Monteiro EMS. Prevalência de transtornos mentais em adolescentes em cumprimento de medida socioeducativa de internação no município do Rio de Janeiro. [dissertação]. Rio de Janeiro: IESC/UFRJ; 2012.

14. Karnik NS, Soller MV, Steiner H. Psychopharmacology and Juvenile Delinquency. In: Kessler CL, Kraus LS, editores. The Mental Health Needs of Young Offenders - Forging Paths toward Reintegration and Rehabilitation. Cambridge: Cambridge University Press, 2007. p. 308-339.

15. Pinho SR, Dunningham W, Aguiar WM, et al. Morbidade psiquiátrica entre adolescentes em conflito com a lei. J. bras. Psiquiatr. 2010; 55(2):126-130.

16. Verugdenhil C, Doreleijers TA, Vermeiren R, et al. Psychiatric disorders in a representative sample of incarcerated boys in the Netherlands. J Am Acad Child Adolesc Psychiatry. 2010, 43(1):97-104.

17. Esbensen F, Peterson D, Taylor TJ, et al. Youth Violence: Sex and Race Differences in Offending, Victimization, and Gang Membership. Philadelphia: Temple University Pres; 2010.

18. Gerring J. Case Selection for Case-Study Analysis. In: Box-Steffensmeier JM, Brady HE, Collier D, editors. The Oxford Handbok of Political Methodology. Oxford: Oxford University Press; 2008. p. 645-684. 
19. Brasil. Superior Tribunal de Justiça. Súmula 492 de 08 de agosto de 2012. [acesso em 2017 abr 9]. Disponível em: http://www.stj. jus.br/SCON/SearchBRS?b=SUMU\&livre=@ docn $=\% 22000000968 \% 22$.

20. Frances A. The Past, Present, and Future of Psychiatric Diagnosis. World Psychiatry, 2013; 12(2):13-18.

21. Zeitoune CM. Gestão em saúde no sistema socioeducativo no Estado do Rio de Janeiro. In: Abdalla JFS, Pereira MBBA, Gonçalves TMT, editores. Ações socioeducativas: estudos e pesquisas. Rio de Janeiro: DEGASE; 2016. p. 37-49.

22. Figueiredo AC, Machado OMR. O Diagnóstico em Psicanálise: Do fenômeno à estrutura. Ágora. 2000 3(2):65-86.

23. Corrado B, Cipriani A, Patel V, et al. Efficacy of antidepressants and benzodiazepines in minor depression: systematic review and meta-analysis. The British Journal of Psychiatry, 2011; (1)98:11-16.

24. Esher A, Coutinho T. Uso racional de medicamentos, farmaceuticalização e usos do metilfenidato. Ciênc. Saúde Colet. 2017; 22(8):2571-2580.
25. Desai RA, Goulet JL, Robbins J, et al. Mental Health Care in Juvenile Detention Facilities: A Review. J Am Acad Psychiatry Law. 2006; (1)34:204-14.

26. Costa NR, Silva PRF. Diagnóstico Situacional da Atenção em Saúde Mental nas Unidades Socioeducativas Brasileiras. Rio de Janeiro: Fiocruz, Ministério da Saúde; 2016

27. Vicentin MCG. A Questão da Responsabilidade Penal Juvenil: Notas para uma Perspectiva Ético-Política. In: Instituto Latino Americano das Nações Unidas para Prevenção do Delito e Tratamento do Delinquente, Associação Baiana do Mercado Publicitário, Secretaria de Estado de Direitos Humanos, et al., editores. Justiça adolescente e Ato Infracional: socioeducação e responsabilização.). São Paulo: ILANUD, 2006. p. $37-45$

Recebido em 28/03/2019

Aprovado em 13/09/2019

Conflito de interesses: inexistente

Suporte financeiro: não houve 